\title{
SuperVIVENCIA DE UNA COHORTE DE PLÁNTULAS DE ABIES RELIGIOSA BAJO DIFERENTES CONDICIONES POSTINCENDIO
}

\author{
Efraín Ángeles-Cervantes ${ }^{1,2}$ y Lauro LÓpez-Mata ${ }^{2,3}$ \\ 'Laboratorio de Ecología de Bosques-Hidrología. Facultad de Estudios Superiores Zaragoza- UNAM. \\ Batalla 5 de mayo s/n esq. Fuerte de Loreto Col. Ejército de Oriente, CP 09230 Iztapalapa, México D. F. \\ ${ }^{2}$ Programa de Botánica, Colegio de Postgraduados, Montecillo, Estado de México. C.P. 56230, México. \\ ${ }_{3}^{3}$ Autor para la correspondencia. Correo-e: lauro@colpos.mx, laurolopezmata@gmail.com
}

\begin{abstract}
Resumen: Se investigó la supervivencia de una cohorte de plántulas de Abies religiosa durante ocho años, en parches de bosque no afectados (BNA) y afectados por incendios: superficiales (BICSUP), de copa moderados (BICMOD) y de copa severos (BICSEV). El objetivo fue aportar mayor entendimiento sobre los procesos de regeneración natural de Abies religiosa. En cada parche se establecieron 8 parcelas de $1 \mathrm{~m}^{2}$ en las que se marcaron las plántulas de la cohorte de 1998. La mortalidad y el tipo de daño asociado se registraron mediante censos desde 1998 hasta 2006. La caída de ramas y la desecación de plántulas fueron los factores más importantes asociados con su muerte. Las probabilidades de supervivencia fueron mínimas en BISUP y nulas en los BNA. Las más altas probabilidades de supervivencia resultaron bajo la condición de BICMOD, lo que sugiere que estos incendios son parte integral del nicho de regeneración de $A$. religiosa.
\end{abstract}

Palabras clave: Coníferas, bosques templados, incendios forestales, regeneración.

\begin{abstract}
The survival of a cohort of Abies religiosa seedlings was investigated during eight years in an intact forest (NAF) and forest patches affected by: shallow fires (SUPAF), intermediate canopy fires (ICAF), and full canopy fires (FCAF). The objective of this paper was to provide a better understanding on the natural regeneration processes of $A$. religiosa. $I n$ each patch eight $1 \mathrm{~m}^{2}$ plots were established and all rooted seedlings within each plot from the 1998 cohort were tagged. Seedling mortality and type of associated damage were recorded throughout censuses from 1998 to 2006 . Fallen branches and desiccation of seedlings were the two most important factors associated with their death. Survival probabilities were the lowest in SUPAF and none in NAF. The highest survival probabilities were under ICAF condition, and it suggests that this type of fire is an important component of the $A$. religiosa regeneration niche.
\end{abstract}

Key Words: Conifers, temperate forests, fires, regeneration.

$\mathbf{L}^{2}$ a supervivencia de plántulas es una de las fases decisivas en el ciclo de vida de las plantas (Harper y White, 1974; Harper, 1977). Los procesos ecológicos y factores ambientales que afectan la supervivencia modifican la dinámica poblacional de la especie e influyen en los atributos estructurales de la comunidad en la cual coexiste (Harcombe, 1987; Gray y Spies, 1996). Su estudio permite entender cómo los procesos naturales intervienen en la regeneración de la población, en especial si la especie está sujeta a disturbios recurrentes. Los incendios forestales son el disturbio natural que más efecto tiene en la dinámica y regeneración natural de los bosques templados (Van Wagner, 1983; BourgueauChavez et al., 2000; Wang, 2002). A pesar de esto, los estudios de supervivencia postincendio de especies de Abies son relativamente escasos y definitivamente no existen estudios de supervivencia de cohortes de plántulas postincendio y menos aún el seguimiento de éstas por varios años. En los bosques de Abies, la regeneración postincendio se ha observado en A. balsamea (L.) Mill. (Heinselman, 1973), A. grandis (Dougl.) Lindl. (Antos y Habeck, 1981), A. lasiocarpa (Hook) Nut. (Agee y Smith, 1984) y A. religiosa $(\mathrm{H}$. B. K.) Schlecht. \& Cham. (Manzanilla, 1976). Estos estudios se han concentrado en la cuantificación de la densidad de plantas acumulada de varias generaciones después de años de haber ocurrido algún incendio. Shatford et al. (2007) evaluaron sitios con $A$. concolor afectados por incendios severos entre 1987 y 1996, registrando densidades que fluctuaron de 0.12 a 1.6 plantas $\mathrm{m}^{-2}$. Estos autores señalan que 
el establecimiento masivo de plántulas se presenta después de tres años, pero desconocen las causas de esta respuesta. Chappell y Agee (1996) estudiaron la regeneración de A. magnifica en bosques afectados por incendios de severidad variable ocurridos en 1978, 1982, 1984, 1986 y 1998. Sus observaciones indican que la máxima densidad de plantas se presentó en sitios afectados por incendios de severidad baja y moderada, mientras que las densidades relativamente bajas se presentaron en sitios con incendios de severidad alta, y densidades mínimas en bosques no afectados $(\approx 10,3$ y 0.5 plantas $\mathrm{m}^{-2}$, respectivamente). Asimismo, la evaluación de la supervivencia de 274 plántulas recién emergidas de $A$. magnifica en los primeros tres meses de edad mostró que el $87 \%$ de la mortalidad era atribuible a la desecación. De acuerdo con Purdy et al. (2002) y Shatford et al. (2007) el proceso de regeneración natural postincendio de coníferas está pobremente documentado. Según Greene et al. (1999) esto se debe a que el reclutamiento postincendio tiene su base en quemas prescritas y de tratamientos del suelo para favorecer la regeneración. Sin embargo, la supervivencia y los factores de mortalidad de plántulas postincendio han originado debates acerca del papel de los incendios sobre la regeneración natural de bosques de coníferas (Baird, 2006; Donato et al., 2006; Newton et al., 2006; Shatford et al., 2007). Tanto DeBano et al. (1998) como Charron y Greene (2002), señalan que algunos bosques de coníferas requieren de incendios para su regeneración por lo que existe la necesidad de investigar sobre el tema y aclarar en qué medida los incendios influencian su regeneración o si se requiere de la implementación de técnicas silvícolas para lograrlo. Por otra parte, Shvidenko y Nilsson (2000) mencionan la dualidad constructiva y destructiva de los incendios. Los incendios destructivos afectan negativamente los procesos de regeneración de las especies y favorecen su exclusión local, mientras que los incendios constructivos favorecen los procesos de reproducción de las especies y la regeneración de los bosques. De aquí se desprende la necesidad de considerar la influencia de los tipos y la severidad de los incendios sobre la supervivencia de plántulas en condiciones postincendio. En México se presentan anualmente, de 6000 a 7000 incendios forestales de diferente intensidad, severidad e intervalo de retorno (Rodríguez et al., 2001), de estos, aproximadamente un $90-96 \%$ corresponde a incendios superficiales, un 4-10\% a incendios de copa y menos de 1\% a incendios subterráneos. En 1998 el número de incendios duplicó la cifra promedio anual y en particular los bosques de Abies religiosa fueron afectados por incendios de diferente tipo y severidad. Por esta razón, la supervivencia de una cohorte de plántulas de A. religiosa emergidas en diferentes condiciones post-incendio en el parque nacional El Chico, Hidalgo, presentó una oportunidad inusual para su estudio. Abies religiosa es una especie nativa de México que forma bosques puros en los que se presentan problemas de regeneración variados y en algunos de ellos una elevada mortalidad de árboles adultos (Alvarado-Rosales y Hernández-Tejeda, 2002).

Los objetivos de esta investigación fueron determinar el impacto que tienen los diferentes tipos y severidad de los incendios en la supervivencia de plántulas de Abies religiosa, y detectar los daños asociados con su mortalidad bajo tres diferentes condiciones postincendio y bajo bosque no afectado. El estudio consistió en el seguimiento durante ocho años de una cohorte de plántulas, desde su emergencia en agosto de 1998 hasta abril de 2006.

\section{Materiales y Métodos}

Área de estudio. Se localiza en el extremo occidental de la sierra de Pachuca, en el parque nacional El Chico, Hidalgo, entre $20^{\circ} 10^{\prime} 10^{\prime \prime}$ y $20^{\circ} 13^{\prime} 25^{\prime}$ " de Latitud Norte y $98^{\circ} 41^{\prime}$ $50^{\prime \prime}$ y $98^{\circ} 46^{\prime} 02^{\prime}$ " de Longitud Oeste, y entre $2320 \mathrm{~m}$ y $3090 \mathrm{~m}$ de altitud. La geología de la zona presenta predominantemente tobas andesíticas, andesitas y riolitas (GalindoLeal et al., 1988). El bosque de Abies religiosa se desarrolla sobre suelos tipo andosol y cambisol con texturas de tipo limoso-arenoso, arcillo-arenoso y arenoso.

El clima es templado semifrío con lluvias en verano, la precipitación media anual es de $1382 \mathrm{~mm}$ y septiembre es el mes con mayor precipitación con un promedio de $302 \mathrm{~mm}$. Las lluvias de invierno representan menos del 5\% de la precipitación anual y las neblinas son frecuentes. De acuerdo con los registros de la estación meteorológica de Mineral del Chico, la precipitación total de los meses secos (noviembre de 1998 a mayo 1999) fue de $11 \mathrm{~mm}$, aproximadamente $50 \%$ menor que el promedio registrado y la precipitación total anual en el año 2000 fue de $688 \mathrm{~mm}$, que representa la mínima registrada en los últimos 60 años. La temperatura media del mes más calido (abril) y del mes más frío (febrero) es de $17{ }^{\circ} \mathrm{C}$ y de $6{ }^{\circ} \mathrm{C}$ respectivamente; aunque se han registrado temperaturas extremas de $38{ }^{\circ} \mathrm{C}$ y de -6 ${ }^{\circ} \mathrm{C}$. La estación seca ocurre de noviembre a mayo, con una precipitación promedio de $21 \mathrm{~mm}$ y en los meses de marzo y abril es común que no se registre precipitación.

En el parque, el bosque de oyamel cubre aproximadamente el $67 \%$ de su superficie cuyo estrato arbóreo esta dominado por Abies religiosa. Las especies dominantes en el estrato arbustivo son Archibaccharis hieracioides Blake, Baccharis conferta H.B.K., Eupatorium hidalgense Rob., Fuchsia thymifolia H.B.K., Ribes affine H.B.K., Salvia elegans Vahl., Senecio angulifolius D.C. y Stevia monardifolia H.B.K. El estrato herbáceo lo dominan, Alchemilla procumbens Rose y Ribes affine H.B.K. y el estrato rasante está dominado por varias especies del musgo Thuidum Schimp. La capa de musgo y hojarasca varía de 3 hasta $25 \mathrm{~cm}$ de espesor.

Sitios de estudio. Durante los meses de marzo y abril de 1998 ocurrieron incendios en el parque los cuales fueron en 
su mayoría de tipo superficial y en menor medida algunos de copa. En julio de 1998, se recorrieron las áreas afectadas por incendios. Se seleccionaron dos sitios cuya extensión incluyó parches de bosque afectado por incendios de diferente severidad. Cada sitio presentó tanto bosque no afectado (BNA), como parches de bosque afectados por incendio superficial (BISUP), afectado por incendio de copa moderado (BICMOD) y afectado por incendio de copa severo (BICSEV) sensu Shividenko y Nilsson (2000). Dadas las circunstancias de los incendios ocurridos en el parque solo fue posible localizar y seleccionar un sitio que presentó incendio de copa severo (BICSEV) mientras que, todas las demás condiciones tuvieron una repetición. En todos los parches afectados, tanto la capa de hojarasca como los estratos rasante, herbáceo y arbustivo fueron consumidos completamente por los incendios. Todos los sitios y parches seleccionados presentaron exposición norte, a una altitud entre los 2930 y 2970 m snm., y distribuidos en suelos tipo andosol.

En los bosques afectados por incendio superficial, todos los árboles con alturas de $2 \mathrm{~m}$ y diámetros a la altura del pecho $(\mathrm{DAP}) \leq 5 \mathrm{~cm}$ murieron; la altura de la flama marcada en el tronco de los árboles fue menor de $1 \mathrm{~m}$. El tamaño de los parches fue de 1.2 (BISUP1) y 0.8 Ha (BISUP2), respectivamente. En los bosques afectados por incendio de copa moderado (BICMOD), todos los árboles con DAP $\leq 30 \mathrm{~cm}$ y alturas $\leq 20 \mathrm{~m}$ murieron. Los árboles sobrevivientes, con alturas de 35 a $40 \mathrm{~m}$ y con DAP entre 35 y $55 \mathrm{~cm}$ presentaron daños en aproximadamente el $75 \%$ de su copa, aunque la marca de la flama sobre el tronco no excedió los $6 \mathrm{~m}$ de altura. El tamaño de éstos parches fue de $0.42 \mathrm{Ha}$ (BICMOD1) y $0.8 \mathrm{Ha}$ (BICMOD2). En el bosque afectado por incendio de copa severo (BICSEV), todos los árboles de todos los tamaños murieron, algunos de ellos en pie. La marca de la flama sobre el tronco alcanzó hasta $30 \mathrm{~m}$ de altura; el tamaño del parche fue de 1.1 Ha. En contraste en los bosques no afectados (BNA), considerados como testigo, no presentaron vestigios de incendios por lo que se conservaron todos los estratos del bosque. El espesor promedio de la hojarasca y el musgo fue de $10 \mathrm{~cm}$.

Cohorte de plántulas. Las semillas de Abies religiosa no forman un banco de semillas en el suelo pues pierden su viabilidad en el mismo año de su dispersión, además de que no se ha registrado depredación de éstas ni consumo total o parcial de sus plántulas por herbívoros (Román, 2002). Esto facilitó iniciar el marcaje y seguimiento inequívoco de plántulas recién emergidas, pertenecientes a la cohorte de 1998, reconocidas además por la presencia de hojas cotiledonares. En cada parche seleccionado se establecieron ocho cuadros de observación permanente de $1 \mathrm{~m}^{2}$. El número de cuadros en cada parche se limitó en función de la abundancia de plántulas. En cada cuadro, se marcó y registró el número de plántulas recién emergidas. A cada plántula se le midió la altura inicial a su ápice y la condición de su estado físico: sana, dañada y tipo de daño para evaluar la causa probable de mortalidad (Fenner, 1987; Fenner y Thompson, 2005). Los tipos de daño considerados fueron: a) herbivoría, b) ataque por hongos, c) desecación o en proceso de desecación y d) daños físicos ocasionados por caída de árboles o ramas, por granizadas y por enterramiento.

Durante el primer año de observaciones se registraron mensualmente tanto la supervivencia como los tipos de daño que pudieran estar asociados a la mortalidad de plántulas. En los siete años siguientes, de 1999 a 2006, los registros se realizaron consistentemente dos veces por año, al final de la estación seca en mayo y al final de la estación húmeda en noviembre.

Análisis de supervivencia. Se seleccionó la función de supervivencia $\mathrm{S}(\mathrm{t})=\operatorname{Pr}\{\mathrm{T}>1\}=1-\mathrm{F}(\mathrm{t})$ que sigue una distribución de mortalidad acumulada $\mathrm{F}(\mathrm{t})$. Esta función de supervivencia es aplicable cuando se desconoce el tiempo exacto de muerte de cualquier plántula y en su lugar estima tanto la probabilidad de supervivencia de una plántula en un intervalo de tiempo, como la función de riesgo de mortalidad dentro del mismo intervalo (Fox, 1993; Allison, 1995). El riesgo de mortalidad está definido por el número de plántulas muertas por intervalo de tiempo, dividido por el número de sobrevivientes en ese intervalo (Fox, 1993). La función de supervivencia y la función de riesgo de mortalidad de las plántulas se obtuvieron con el método de tablas de vida, mediante el procedimiento LIFETEST (SAS, 1998). LIFETEST estima las probabilidades de supervivencia y de riesgo para datos que proporcionen: a) los límites de los intervalos; b) el número de muertes en cada intervalo; y c) el número de casos censados en cada intervalo. Este procedimiento permite, además, comparar pares de curvas de supervivencia y detectar si existen diferencias estadísticamente significativas entre ellas y probar la hipótesis nula de que la función de supervivencia es idéntica entre pares de curvas (Fox, 1993; Allison, 1995).

El tipo de daño en las plántulas se evaluó con un modelo de regresión de máxima verosimilitud, utilizando el procedimiento LIFEREG (SAS, 1998). LIFEREG proporciona modelos de regresión para datos tomados a intervalos periódicos de tiempo y el análisis de variables categóricas con valores múltiples, por lo que es posible probar la asociación del tipo de daño con la mortalidad de plántulas (Allison, 1995). Para mejorar la estimación de los coeficientes de regresión se determinó el tipo de distribución de la función de la supervivencia bajo cada condición postincendio, por comparación con las distribuciones teóricas Weibull, gamma, exponencial, log-normal y log-logística (Allison, 1995).

\section{Resultados}

Densidad de plántulas. Se registraron en total 1434 plántulas; la densidad inicial de plántulas en cada parche fue 


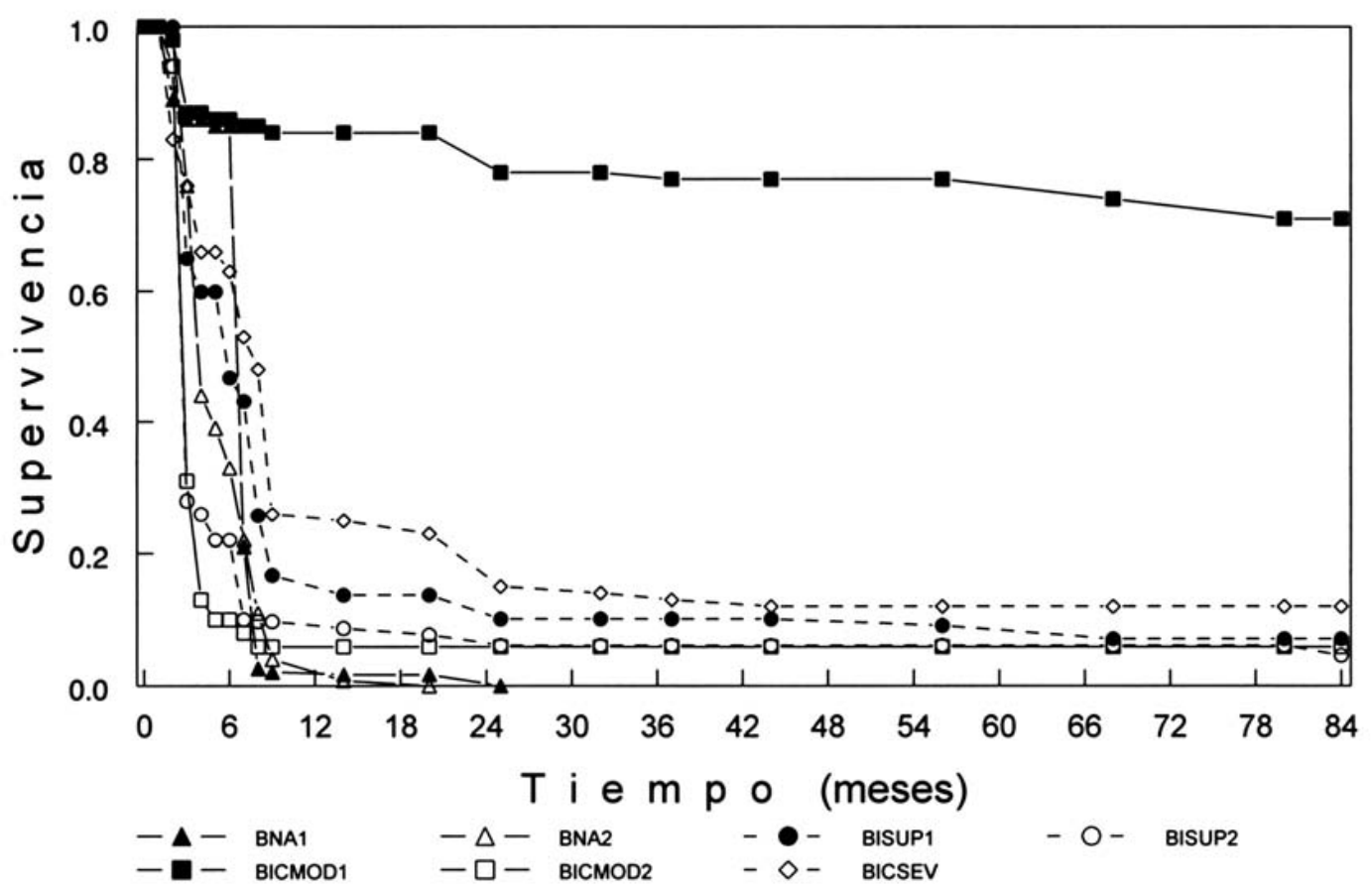

Figura 1. Curvas de supervivencia de la cohorte de plántulas de Abies religiosa bajo condiciones de bosque no afectados por incendios (BNA), afectados por incendios superficiales (BISUP), afectados por incendios de copa moderados (BICMOD) y afectados por incendio de copa severo (BICSEV).

de $28.5 \pm 9.4$ (media \pm error estándar), $30.4 \pm 17.5,24.6 \pm 17.9$, $24.2 \pm 12.2,26.7 \pm 8.3,23.1 \pm 12.6$ y $22.1 \pm 6.0$, para el BNA1, BNA2, BISUP1, BISUP2, BICMOD1, BICMOD2 y BIC$\mathrm{SEV}$, respectivamente. Sin embargo un análisis de varianza no mostró diferencias significativas entre ellos $(\mathrm{F}=0.44$, g.l. $=6, \mathrm{p}>0.85)$. El número remanente de plántulas al final del estudio fue de 166. La densidad residual promedio de plántulas fue de $16.5 \pm 5.9$ en el BICMOD1, seguidas del BICSEV y BICMOD2 con $2.6 \pm 1.8$ y $1.4 \pm 0.7$, respectivamente; en tanto que en el BISUP1 y BISUP2 se presentaron densidades menores de $1(0.62 \pm 1.0$ y $0.12 \pm 0.3$, respectivamente). En contraste, en los bosques no afectados, BNA2 y BNA1, la densidad de plántulas fue cero a partir de los 20 y 24 meses de edad, respectivamente.

Supervivencia y riesgos de mortalidad. La figura 1 muestra las curvas de supervivencias de plántulas de Abies religiosa. Fue evidente que, con excepción de la curva bajo la condición BICMOD1, las curvas de supervivencia de $A$. religiosa pertenecen al tipo III (Pearl y Miner, 1935) y muestra que la probabilidad de supervivencia disminuyó drásticamente en los primeros doce meses, y en menor medida hasta los veinticuatro meses, donde las probabilidades de supervivencia fueron de 0.24, 0.08, 0.07 y 0.06, para BICSEV, BISUP1, BISUP2 y BICMOD2 respectivamente. Las probabilidades de supervivencia de las plántulas remanentes en los parches afectados permanecieron relativamente constantes durante los 60 meses restantes (figura 1). El caso extremo de supervivencia nula, durante los 20 y 24 meses de edad se presentó en los sitios que no fueron afectados por incendios (BNA2 y BNA1, respectivamente).

Sorprendentemente, las plántulas bajo la condición de incendio de copa moderado (BICMOD1) presentaron una curva de supervivencia que se distingue de todas las anteriores por presentar una elevada probabilidad de supervivencia. Esta fluctuó de 0.84 durante los primeros 12 meses de edad hasta 0.74 durante los seis años restantes (figura 1). Bajo esta condición, el porcentaje de mortalidad de plántulas durante los primeros 12 meses de edad fue de $16 \% \quad(n=34)$, mientras que durante los restantes 70 meses fue de tan solo el $4.5 \%(n=8)$ en promedio (figura 1). La comparación entre pares de curvas mostró que sólo las curvas de supervivencia en el BISUP2 y BICMOD2 resultaron homogéneas entre sí, mientras que, todos los pares de curvas remanentes, resultaron heterogéneas (cuadro 1).

Esto sugiere que la supervivencia de plántulas de Abies religiosa denota una respuesta diferencial a las condiciones de bosque estudiadas. En la figura 2, se muestran los riesgos de mortalidad de plántulas, en bosques afectados y no afectados por incendios, en función de su edad. En ambos bosques, los más altos riesgos de mortalidad se presentaron consistentemente durante los primeros 10-14 meses de edad. En los bosques no afectados, los riesgos de mortalidad fueron los más altos (1.6), mientras que en los bosque afectados fluctuaron entre 0.11 en el BICMOD1 y 1.1 en el BISUP2 (figura 2). 
Cuadro 1. Matriz de comparación entre pares de curvas de supervivencia de A. religiosa bajo condiciones de bosque afectado y no afectado por incendios en el parque Nacional El Chico, Hidalgo. Los valores de $\chi^{2}$ corresponden a la prueba Log-Rank; los grados de libertad en todos los casos es igual a 1. BNA = bosque no afectado; BISUP = bosque afectado por incendio superficial; $\mathrm{BICMOD}=$ bosque afectado por incendio de copa moderado y BICSEV = bosque afectado por incendio de copa severo.

\begin{tabular}{|c|c|c|c|c|c|c|c|c|}
\hline & \multicolumn{2}{|c|}{ BNA1 } & $\mathrm{BNA} 2$ & BISUP1 & \multicolumn{2}{|c|}{ BISUP2 } & BICMOD1 & BICMOD2 \\
\hline & $\chi^{2}$ & $P$ & $\chi^{2}$ & $\chi^{2}$ & $\chi^{2}$ & $\mathrm{P}$ & $\chi^{2}$ & $\chi^{2}$ \\
\hline BNA2 & 22.84 & $<0.0001$ & & & & & & \\
\hline BISUP1 & 4.96 & 0.0260 & $24.97<0.0001$ & & & & & \\
\hline BISUP2 & 46.64 & $<0.0001$ & $8.11 \quad 0.004$ & $31.53<0.0001$ & & & & \\
\hline BICMOD1 & 278.40 & $<0.0001$ & $332.30<0.0001$ & $239.11<0.0001$ & 297.59 & $<0.0001$ & & \\
\hline BICMOD2 & 98.31 & $<0.0001$ & $30.66<0.0001$ & $38.85<0.0001$ & 0.009 & 0.924 & $239.36<0.0001$ & \\
\hline BICSEV & 40.39 & $<0.0001$ & $64.83<0.0001$ & $10.79<0.0001$ & 51.56 & $<0.0001$ & $145.19<0.0001$ & $57.36<0.0001$ \\
\hline
\end{tabular}

Tipos de daño. El cuadro 2 muestra los efectos significativos de los distintos tipos de daño sobre la supervivencia de las plántulas. Los tipos de daño que consistentemente afectaron la supervivencia de las plántulas bajo cualquier condición fueron: la caída de ramas y la desecación progresiva probablemente asociada al estrés hídrico. En cambio, los daños ocasionados por la caída de árboles dañados o muertos en pié, afectaron la supervivencia de plántulas solamente en el bosque afectado por incendio de copa severo (BICSEV). También, en los bosques afectados por incendios superficiales y de copa severo (BISUP1, BISUP2 y BICSEV), la mortalidad de plántulas se asoció significativamente al ataque por hongos patógenos. Finalmente, en los bosques con incendios superficiales (BISUP2) y de copa moderado (BICMOD2), la muerte de $60 \%$ y $63 \%$ de plántulas se asoció con su enterramiento durante las lluvias torrenciales de septiembre, respectivamente.

\section{Discusión}

Las curvas de supervivencia de la cohorte de plántulas de Abies religiosa se ajustan a las curvas tipo I y tipo III propuestas por Pearl y Miner (1935). La curva tipo III la presentan también otras especies de Abies, como A. vietchii y A. mariesii (Kohyama y Fujita, 1981), A. lasiocarpa (Noble y Ronco, 1978), A. concolor y A. magnifica (Barbour et $a l .$, 1990). En tanto que la curva tipo I no ha sido documentada para cohortes de especies de coníferas arbóreas. Sin embargo, tanto Shatford et al. (2007) como Chappell y Agee (1996) y Waldrop y Brose (1999) reportaron alta regeneración postincendio en Abies concolor, A. magnifica y Pinus pungens, respectivamente. Las curvas de supervivencia tipo I y tipo III contrastan con la curva tipo II, observada por Hett (1971) y Hett y Loucks (1976) en plántulas de Acer saccharum y Abies balsamea, respectivamente.

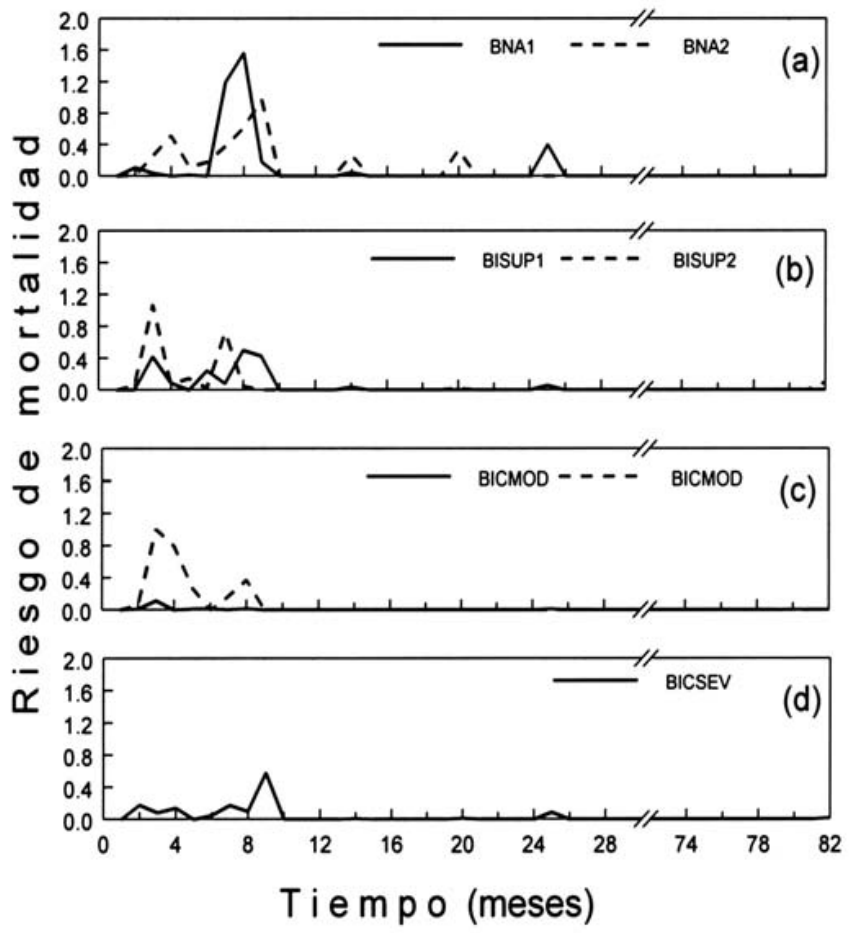

Figura 2. Riesgos de mortalidad de plántulas de A. religiosa en: (a) bosques no afectados por incendios (BNA1 y BNA2), (b) afectados por incendios superficiales (BISUP1 y BISUP2), (c) afectados por incendios de copa moderados (BICMOD1 y BICMOD2) y (d) afectado por incendio de copa severo (BICSEV).

Por otra parte, el alto riesgo de mortalidad y nula supervivencia de plántulas de Abies religiosa, en los bosques no afectados, sugieren que la regeneración en estos bosques se favorece en respuesta a disturbios ocasionados por incendios. Una de las consecuencias de disturbios en los bosques 
Cuadro 2. Factores de mortalidad de plántulas de Abies religiosa en bosques afectados y no afectados por incendios. Se presenta el coeficiente de regresión $(\beta) \pm$ E.E. (error estándar) para cada condición de bosque. BNA = bosques no afectados, BISUP = bosques afectados por incendios superficiales; BICMOD = bosques afectados por incendios de copa moderados y BICSEV = bosque afectado por incendio de copa severo.

\begin{tabular}{|c|c|c|c|c|c|}
\hline Condición & Variable & g.l. & $\beta \pm \mathrm{E} . \mathrm{E}$. & $\chi^{2}$ & $\mathrm{P}$ \\
\hline \multirow[t]{4}{*}{ BNA1 } & Intercepto & 1 & $0.693 \pm 0.029$ & 545.75 & 0.0001 \\
\hline & Factor de mortalidad & 3 & & 10208.71 & $<0.0001$ \\
\hline & Caída de ramas & 1 & $-0.687 \pm 0.034$ & 398.68 & 0.0001 \\
\hline & Desecación & 1 & $1.128 \pm 0.030$ & 1373.35 & 0.0001 \\
\hline \multirow[t]{4}{*}{ BNA2 } & Intercepto & 1 & $1.152 \pm 0.014$ & 6521.14 & $<0.0001$ \\
\hline & Factor de mortalidad & 3 & & 3019.69 & $<0.0001$ \\
\hline & Caída de ramas & 1 & $-0.468 \pm 0.021$ & 471.94 & $<0.0001$ \\
\hline & Desecación & 1 & $0.793 \pm 0.022$ & 1307.27 & $<0.0001$ \\
\hline \multirow[t]{5}{*}{ BISUP1 } & Intercepto & 1 & $4.512 \pm 0.082$ & 3000.92 & $<0.0001$ \\
\hline & Factor de mortalidad & 3 & & 61.80 & $<0.0001$ \\
\hline & Caída de ramas & 1 & $-3.818 \pm 0.087$ & 1903.59 & $<0.0001$ \\
\hline & Desecación & 1 & $-2.551 \pm 0.087$ & 855.47 & $<0.0001$ \\
\hline & Hongos & 1 & $-3.413 \pm 0.110$ & 954.53 & $<0.0001$ \\
\hline \multirow[t]{6}{*}{ BISUP2 } & Intercepto & 1 & $4.453 \pm 0.061$ & 5280.12 & $<0.0001$ \\
\hline & Factor de mortalidad & 4 & & 4891.54 & $<0.0001$ \\
\hline & Caída de ramas & 1 & $-4.454 \pm 0.075$ & 3452.33 & $<0.0001$ \\
\hline & Enterramiento & 1 & $-3.760 \pm 0.062$ & 3600.76 & $<0.0001$ \\
\hline & Desecación & 1 & $-2.552 \pm 0.070$ & 1327.87 & $<0.0001$ \\
\hline & Hongos & 1 & $-3.160 \pm 0.081$ & 1506.71 & $<0.0001$ \\
\hline \multirow[t]{6}{*}{ BICMOD1 } & Intercepto & 1 & $12.966 \pm 27893.8$ & 0.00 & n.s \\
\hline & Factor de mortalidad & 4 & & 853.30 & $<0.0001$ \\
\hline & Caída de ramas & 1 & $-12.335 \pm 27893.8$ & 0.00 & n.s. \\
\hline & Caída de árboles & 1 & $-8.940 \pm 27893.8$ & 0.00 & n.s. \\
\hline & Ramoneo de tallo & 1 & $-9.970 \pm 27893.8$ & 0.00 & n.s. \\
\hline & Desecación & 1 & $-8.848 \pm 27893.8$ & 0.00 & n.s. \\
\hline \multirow[t]{5}{*}{ BICMOD2 } & Intercepto & 1 & $5.014 \pm 0.001$ & 27700036 & $<0.0001$ \\
\hline & Factor de mortalidad & 3 & & 16895401.9 & $<0.0001$ \\
\hline & Caída de ramas & 1 & $-5.014 \pm 0.001$ & 9999.9 & $<0.0001$ \\
\hline & Enterramiento & 1 & $-4.321 \pm 0.001$ & 9999.9 & $<0.0001$ \\
\hline & Desecación & 1 & $-3.069 \pm 0.002$ & 9999.9 & $<0.0001$ \\
\hline \multirow[t]{6}{*}{ BICSEV } & Intercepto & 1 & $4.418 \pm 0.006$ & 425320 & $<0.0001$ \\
\hline & Factor de mortalidad & 4 & & 21752585.3 & $<0.0001$ \\
\hline & Caída de ramas & 1 & $-4.418 \pm 0.007$ & 9999.9 & $<0.0001$ \\
\hline & Caída de árboles & 1 & $-1.423 \pm 0.007$ & 9999.9 & $<0.0001$ \\
\hline & Desecación & 1 & $-2.627 \pm 0.007$ & 9999.9 & $<0.0001$ \\
\hline & Hongos & 1 & $-3.320 \pm 0.008$ & 9999.9 & $<0.0001$ \\
\hline
\end{tabular}

son las respuestas de las plantas a la generación de claros de luz de tamaños, formas, orientación y aspecto variables (Canham y Marks, 1986; Veblen, 1989). Sin embargo, la penetración de la luz a través de la heterogeneidad física de los claros no está plenamente entendida en el género Abies. Los trabajos con Abies grandis (Antos y Habeck, 1981), A. lasiocarpa (Knapp y Smith, 1982; Agee y Smith, 1984) y A. magnifica (Scholl y Taylor, 2006), documentaron una regeneración exitosa tanto a la sombra como en claros de luz. En nuestra investigación, las densidades máximas de plántulas resultaron en los claros ocasionados por incendios de copa moderados, lo que fortalece la relevancia de los incendios sobre la regeneración del bosque. Sin embargo, una investigación de la supervivencia de plántulas en claros ocasionados por la caída de árboles, per se, permitirá entender en que medida estos influencian la regeneración del bosque. Asimismo, con excepción de la condición de incendio de copa moderado (BICMOD1), bajo todas las condiciones estudiadas y durante los primeros 10 a 14 meses de edad, las plántulas experimentaron su mortalidad máxima. Durante estos primeros meses los riesgos de mortalidad fueron los más altos e importantes durante su fase de establecimiento. Durante esos primeros meses, la presencia de los estratos herbáceo y arbustivo fue escasa en todos los sitios incendiados y nulos en los cuadros de muestreo, pero ampliaron su cobertura con el tiempo. Sin embargo, el impacto de su 
potencial competencia con las plántulas de A. religiosa fue despreciable ya que la supervivencia de éstas permaneció relativamente constante en cada condición durante los 70 meses siguientes. En los parches de bosque que presentaron una curva de supervivencia tipo III, la elevada mortalidad de plántulas en los primeros meses de edad se asoció con la caída de ramas. Este factor fue el denominador común y el de mayor impacto en la supervivencia de las plántulas tanto en bosques afectados como en los no afectados por incendios. Las fuertes lluvias y vientos asociados durante el segundo mes de estudio (septiembre), promovieron la caída de ramas y de árboles muertos en pie. La importancia e impacto sobresaliente de la caída de ramas en la supervivencia de plántulas de $A$. religiosa explica, parcialmente, su elevada mortalidad en los primeros meses de vida. La caída de ramas se presentó en una sola ocasión, tanto en los bosques no afectados como los afectados por incendio superficial. En contraste, la caída de ramas en el bosque con incendio de copa severo se presentó en varios episodios y en ocasiones de manera relativamente continua. En cambio, en el bosque con incendio de copa moderado las ramas persistieron en el árbol hasta por cuatro años lo que implicó que sus efectos en la supervivencia de plántulas fueran insignificantes.

Por otra parte, la desecación de plántulas fue el segundo factor en importancia que impactó significativamente su supervivencia. En los bosques no afectados, la desecación de plántulas es atribuible a la capa de musgo y hojarasca acumulada, la cual impide a la raíz penetrar y fijarse al suelo ( $\mathrm{Si}$ mard et al., 2003). En esta capa se ha detectado la emisión de sustancias alelopáticas (Rice, 1979; Rizvi y Rizvi, 1992), y la formación de compuestos hidrófobos en ella (DeBano et al., 1998). En cambio, en los bosques afectados por incendios superficiales (BISUP), la excesiva resequedad del suelo sugiere que la desecación de plántulas puede estar asociada con la generación de capas hidrófobas en el suelo. La combustión incompleta de materia orgánica a temperaturas entre 80 y $175^{\circ} \mathrm{C}$ generan la presencia de compuestos hidrófobos (DeBano et al., 1998). Santander (2004) detectó la presencia de compuestos hidrófobos en los parches de BISUP1 y BISUP2.

En los bosques afectados por incendios de copa severos, la desecación de las plántulas se atribuye a los cambios drásticos en la estructura del suelo y, en consecuencia, a la disminución de la porosidad y capacidad para almacenar agua. De acuerdo con Giovannini y Lucchesi (1997), estos cambios se presentan en suelos que han sido sometidos a temperaturas mayores de $460^{\circ} \mathrm{C}$. Sin embargo, Santander (2004) no encontró compuestos hidrófobos generados en la condición de BICSEV. Además, es probable que la desecación de plántulas durante el primer año de estudio, se haya magnificado debido a la extraordinaria sequía que se presentó durante la estación seca (noviembre a mayo) de ese año. En contraste, la desecación no ocasionó daños significativos de plántulas en los bosques afectados por incen- dios de copa moderados (BICMOD). En estos bosques, la supervivencia de plántulas fue extremadamente elevada, lo que sugiere que este tipo de incendios tiende a favorecer su establecimiento y permanencia a largo plazo. Tal respuesta se atribuye a que las temperaturas alcanzadas en este tipo de incendio destruyen los compuestos hidrófobos permitiendo con ello una mayor disponibilidad de agua. Santander (2004) no detectó la presencia de compuestos hidrófobos en suelos provenientes de BICMOD1 y BICMOD2.

La elevada mortalidad de plántulas en BISUP2 y BICMOD2 generó la homogeneidad en sus curvas de supervivencia. Las causas se debieron al efecto combinado del enterramiento de las plántulas por el deslizamiento del suelo superficial magnificado en pendientes mayores a $40 \%$ prevalecientes en ambas condiciones. La muerte asociada con el ataque por hongos es atribuible a la caída de ramas infectadas que los transmiten a las plántulas tanto en el BISUP1 y BISUP2, como en el BICSEV. Shafizadeh y Kavanagh (2005) mostraron que las plántulas de Abies son más susceptibles al ataque por hongos que otras coníferas; mientras que la transmisión por ramas infectadas han sido documentadas por Fox (1977) y por Girson y Salinas (1985). Por último la caída de árboles en BICSEV, se atribuye a que el árbol muerto en pie es más susceptible a caer por la incidencia de vientos fuertes. Los incendios superficiales son el tipo de incendios más frecuente en México y muy nocivos para la regeneración natural de Abies religiosa ya que bajo estas condiciones, las probabilidades de supervivencia son mínimas. Nosotros sugerimos, investigar más detalladamente sus efectos sobre las propiedades hidrológicas y químicas del suelo y postulamos que sus efectos podrían ser la causa principal de mortalidad masiva de plántulas. Similarmente, los incendios de copa severos no son adecuados para la regeneración natural de $A$. religiosa porque al destruirse todo el arbolado adulto en un área dada, el reclutamiento local de plántulas postincendio no se garantiza. En cambio, la elevada supervivencia de las plántulas en el bosque con incendios de copa moderado (BICMOD1) sugiere que éstos juegan el papel más importante en la promoción de la regeneración natural del bosque de Abies religiosa. Los tres tipos de incendios estudiados y su impacto en la generación diferencial de condiciones edáficas locales determinaron las condiciones ambientales que toleran las plántulas de esta especie. Nuestros resultados sugieren que las condiciones edáficas generadas por los incendios de copa moderados son parte integral del nicho de regeneración, sensu Grubb (1977), de Abies religiosa.

\section{Agradecimientos}

El primer autor agradece al CONACYT el apoyo por cuatro años, mediante la beca 181515, para realizar sus estudios doctorales. La Universidad Nacional Autónoma de México apoyó con recursos materiales y económicos de la DGAPA- 
UNAM y la FES Zaragoza-UNAM, a través de los proyectos PAPIIT- IN217300 y PAPITT- IN200603. L. LópezMata agradece al CONACYT el otorgamiento de una beca por un año sabático el cual hizo posible finalizar este artículo. Los datos climatológicos de la estación meteorológica del Mineral del Chico, Hidalgo, fueron proporcionados amablemente por la Comisión Nacional del Agua, Delegación Pachuca, Estado de Hidalgo. El Ing. Celso Lazcano Cázarez, de la SEMARNAT-Hidalgo, otorgó amplias facilidades para la investigación dentro del parque. Apreciamos y agradecemos enormemente las revisiones críticas, sugerencias y recomendaciones de dos árbitros anónimos, así como los comentarios de la editora, los cuales permitieron mejorar sustancialmente el manuscrito. L. López-Mata agradece al CONACyT su beca sábatica.

\section{Literatura citada}

Agee J.K. y Smith L. 1984. Subalpine tree reestablishment after fire in the Olympic Mountains, Washington. Ecology 65:810-819.

Allison P. 1995. Survival analysis using the SAS@ system. A practical guide. SAS Institute Inc. Cary, North Carolina.

Alvarado-Rosales D. y Hernández-Tejeda T. 2002. Decline of sacred fir in the Desierto de los Leones national park. En: Fenn M.E., de Bauer L.I. y Hernández-Tejeda T. Eds. Urban air pollution and forests: resources at risk in the Mexico city air basin, pp.122-159. Springer-Verlag, New York.

Antos J.A. y Habeck J.R. 1981. Successional development in Abies grandis (Dougl.) Forbes forests in the Swan Valley, Western Montana. Northwest Science 55:26-39.

Baird B.N. 2006. Comment on post-wildfire logging hinders regeneration and increases fire risk. Science 313:615b.

Barbour M.G., Pavlik B.M. y Antos J.A. 1990. Seedling growth and survival of red and white fir in a Sierra Nevada ecotone. American Journal of Botany 77:927-938.

Bourgueau-Chavez L., Alexander M.E., Stocks B.J. y Kasischke E.S. 2000. Distribution of forest ecosystems and the role of fire in the North American boreal region. En: Kasischke E.S. y Stocks B.J. Eds. Fire, Climate Change, and Carbon Cycling in the Boreal Forest, pp. 111-131, Springer- Verlag, New York.

Canham C.D. y Marks P.L. 1986. The response of woody plants to disturbance: patterns of establishment and growth. En: Pickett S.T.A. y White P.S. Eds. The ecology of natural disturbance and patch dynamics, pp. 197-216, Academic Press, Orlando.

Chappell C.B. y Agee J.K. 1996. Fire severity and tree seedling establishment in Abies magnifica forests, southern cascades Oregon. Ecological Applications 6:628-640.

Charron I. y Greene D.F. 2002. Post-wildfire seedbeds and tree establishment in the southern mixewood boreal forest. Canadian Journal of Forest Research 32:1607-1615.

DeBano L.F., Neary D.G. y Ffolliott P.F. 1998. Fire's effects on Ecosystems. John Wiley \& Sons. New York.

Donato D.C., Fontaine J.B., Campbell J.L., Robinson W.D., Kauffman J.B. y Law B.E. 2006. Post-wildfire logging hinders regeneration and increases fire risk. Science 311:352.

Fenner M. 1987. Seedlings. New Phytologist 106 (suppl.): 35-47.

Fenner M. y Thompson K. 2005. The ecology of seeds. Cambridge University Press, Cambridge UK.
Fox J.F. 1977. Alternation and coexistence of tree species. The American Naturalist 111:69-89.

Fox G.P. 1993. Failure-time analysis: Emergence, flowering, survivorship, and other waiting times. En: Scheiner S.M. y Gurevitch J. Eds. Design and Analysis of Ecological Experiments, pp. 253-289, Chapman Hall. New York.

Galindo-Leal C., Franco I.S., Flores M. A, Lazcano C. y Cortés R. 1988. Plan de manejo del parque nacional El Chico. Secretaría de Desarrollo Urbano y Ecología. Delegación Hidalgo. México.

Girson I.A. y Salinas R. 1985. Notas sobre enfermedades forestales y su manejo. Boletín Técnico 106. Instituto Nacional de Investigaciones Forestales. México.

Giovannini G. y Lucchesi S. 1997. Modifications induced in soil physico-chemical parameters by experimental fires at different intensities. Soil Science 162:479-486.

Gray A.N. y Spies T. A. 1996. Gap size, within-gap position and canopy structure effects on conifer seedling establishment. The Journal of Ecology 84:635-645.

Greene D.F., Zasada J.H., Sirois L., Kneeshaw D., Morin H., Charron L. y Simard M-J. 1999. A review of the regeneration dynamics of North American boreal forest tree species. Canadian Journal of Forest Research 29:824-839.

Grubb P.J. 1977. The maintenance of species-richness in plant communities: the importance of the regeneration niche. Biological Reviews 52:107-145.

Harcombe P.A. 1987. Tree life tables. BioScience 37:557-568.

Harper J.L. 1977. Population Biology of Plants. Academic Press. Orlando.

Harper J.L. y White J. 1974. The demography of plants. Annual Review of Ecology and Systematics 5:419-463.

Heinselman M. L. 1973. Fire in the virgin forests of the Boundary Waters Canoe Area, Minnesota. Quaternary Research 3:329382.

Hett J. 1971. A dynamic analysis of age in sugar maple seedlings. Ecology 52:1071-1074.

Hett J. y Loucks O.L. 1976. Age structure models of Balsam fir and Eastern Hemlock. The Journal of Ecology 64:1029-1044.

Knapp A.K. y Smith W.K. 1982. Factors influencing understory seedling establishment of Engelmann spruce (Picea engelmannii) and subalpine fir (Abies lasiocarpa) in southeast Wyoming. Canadian Journal of Botany 60:2753-2761.

Kohyama T. y Fujita N. 1981. Studies on the Abies population of Mt. Shimagare. I. survivorship curve. Botanical Magazine Tokyo 94:55-68.

Manzanilla H. 1976. Investigaciones epidiométricas y silvícolas en bosques mexicanos de Abies religiosa. SAG. México.

Newton M., Fitzgerald S., Rose R.R., Adams P.W., Tesch S.D., Sessions J., Atzet T., Powers R.F. y Skinner C. 2006. Comment on post-wildfire logging hinders regeneration and increases fire risk. Science 313:615a.

Noble D. y Ronco F. 1978. Seed fall and establishment of Engelman spruce and Subalpine fir in clearcut openings in Colorado. USDA. Forest Service Research. Paper RM 200. Rocky Mountain Forest And Range Experimental. 12 pp.

Pearl R. y Miner J.R. 1935. Experimental studies on the duration of life. XIV. The comparative mortality of certain lower organisms. The Quarterly Review of Biology 10: 60-79.

Purdy B.G., Macdonald S.E. y Dale M.R.T. 2002. The regeneration niche of white spruce following fire in the mixed-wood boreal forest. Silva Fennica 36:289-306 
Rice E.L. 1979. Allelopathy-An update. The Botanical Review 45:15-109.

Rizvi S.J.H. y Rizvi V. 1992. Allelopathy. Basic and applied aspects. Chapman Hall. London.

Rodríguez T.D.A., Rodriguez A.M., Fernández S.F. y Pyne S.J. 2001. Educación e incendios forestales. Ed. Mundi-prensa. México.

Román I.R. 2002. Ecología de semillas y plántulas de Abies religiosa (HBK) Schl. et Cham. en el parque nacional "Cumbres del Ajusco", D. F., México. Tesis de Licenciatura, Facultad de Estudios Superiores Zaragoza, Universidad Nacional Autónoma de México. México D. F. 50 pp.

Rzedowski J. 1978. La vegetación de México. Ed. Limusa. México.

Santander M. 2004. Caracterización de la materia orgánica por espectroscopia de infrarrojo (FT-IR) y su relacion con nitrógeno y fósforo en suelo postincendio de un bosque de oyamel. Tesis de Licenciatura, Facultad de Estudios Superiores Zaragoza, Universidad Nacional Autónoma de México. México D. F. 111 pp.

SAS. 1998. SAS/STAT User Guide, V6. Fourth Edition, Volume 2. SAS Institute Inc. Cary. North Carolina.

Scholl A.E. y Taylor A.H. 2006. Regeneration patterns in oldgrowth red fir-western white pine forests in a northern Sierra Nevada, Lake Tahoe, USA. Forest Ecology and Management 235:143-154.

Shafizadeh S. y Kavanagh J.A. 2005. Pathogenicity of Phytoph- thora species and Pythium undulatum isolated from Abies procera Christmas trees in Ireland. Forest Pathology 35:444-450.

Shatford J.P.A., Hibbs D.E. y Puettmann K.J. 2007. Conifer regeneration after forest fire in the Klamath-Siskiyous: How much, How soon? Journal of Forestry 105:139-146.

Shvidenko A.Z. y Nilsson S. 2000. Extent, distribution, and ecological role of fire in Russian forest. En: Kasischke E. y Stocks B.J. Eds. Fire, climate change, and Carbon cycling in the boreal forest, pp. 132-150, Springer-Verlag, New York.

Simard M.J., Bergeron Y. y Sirois L. 2003. Substrate and litterfall effects on conifer seedling survivorship in southern boreal stands of Canada. Canadian Journal of Forest Research 33:672-681.

Van Wagner C. E. 1983. Fire behavior in northern coniferous forests. En: Wein R.W. and MacLean D.A. Eds. The role of fire in northern circumpolar ecosystems, pp. 65-80. John Wiley \& Sons, New York.

Veblen T.T. 1989. Tree regeneration responses to gaps along a transandean gradient. Ecology 70:541-543.

Waldrop T. y Brose P.H. 1999. A comparison of fire intensity levels for stand replacement of table mountain pine (Pinus pungens Lamb.). Forest Ecology and Management 113:155-166.

Wang G.G. 2002. Fire severity in relation to canopy composition within burned boreal mixedwood stands. Forest Ecology and Management 163:85-92. 